\title{
Dark Energy and the Entropy of the Observable Universe
}

\author{
Charles H. Lineweaver ${ }^{\mathrm{a}}$ and Chas A. Egan ${ }^{\mathrm{b}}$ \\ ${ }^{a}$ Planetary Scinece Institute, Research School of Astronomy and Astrophysics, and Research School of Earth \\ Sciences Australian National University \\ ${ }^{b}$ Research School of Astronomy and Astrophysics, Australian National University
}

\begin{abstract}
The initial low entropy of the universe has allowed irreversible processes, such as the reader reading this abstract, to happen in the universe. This initial low entropy is due to a low value for the initial gravitational entropy of the universe. The standard $\Lambda \mathrm{CDM}$ cosmology has a cosmic event horizon and an associated Gibbons-Hawking entropy. We compute the entropy of the universe including the entropy of the current event horizon and the entropy of the matter and photons within the cosmic event horizon. We estimate the entropy of the current cosmic event horizon to be $2.6 \pm$ $0.3 \times 10^{122} \mathrm{k}$ and find it to be $\sim 10^{19}$ times larger than the next most dominant contribution, which is from super massive black holes. We plot an entropy budget as a function of time and find that the cosmic event horizon entropy has dominated other sources of entropy since $10^{-20}$ seconds after the big bang. See Egan \& Lineweaver (2009) for details and discussion.
\end{abstract}

Keywords: Entropy, Gravity, Dark Energy

PACS: 96.10, 96.12, 96.55, 98.80

\section{THE INITIAL ENTROPY PROBLEM OF THE UNIVERSE}

Irreversible things keep happening because the universe is not in equilibrium. Coffee cups keep cooling down, the Sun keeps shining and we all keep going about our business oxidizing carbohydrates, then dying. The second law of thermdynamics, dS $\geqq 0$, quantifies these irreversible changes and provides a direction to time. If the universe had started out in equilibrium, the entropy of the universe, $S_{\text {uni }}$, would be a maximum; $S_{\text {uni }}=S_{\max }$, and irreversible processes, like cooling coffee cups and life forms, would not happen. Without irreversible processes we would have $\mathrm{dS}=0$, and this fact would be unobservable since there would be no irreversible processes to observe it.

The early universe appears to have been in thermal and chemical equilibrium. Figure 1 is an image of the cosmic microwave background (CMB) and shows the universe as it was about 380,000 years after the big bang. In any one direction, the temperature of the photosphere of the universe, (i.e. the surface of last scattering) is almost identical to the temperature in the opposite direction. Both are $\sim 2.725 \mathrm{~K}$ (Mather et al 1999). Variations in the temperature of the CMB are a few parts in $10^{5}$ (Smoot et al 1992) and are too small to be seen on this map. If the early universe were in complete thermal, chemical (and all other types of) equilibrium, it would have been at maximum entropy. Therefore, some low entropy component must be missing, otherwise the entropy of the universe could not have increased as it has over the past 13.7 billion years. Thus, although the CMB appears to suggest that $S_{\text {initial }} \sim S_{\max }$, we must have $S_{\text {initial }}<<S_{\text {now }}<<$ $S_{\max }$. Penrose $(1979,1987,1989 \& 2004)$ has pointed out the significance of this paradox and has suggested that low gravitational entropy (Fig. 2) is the missing component. 


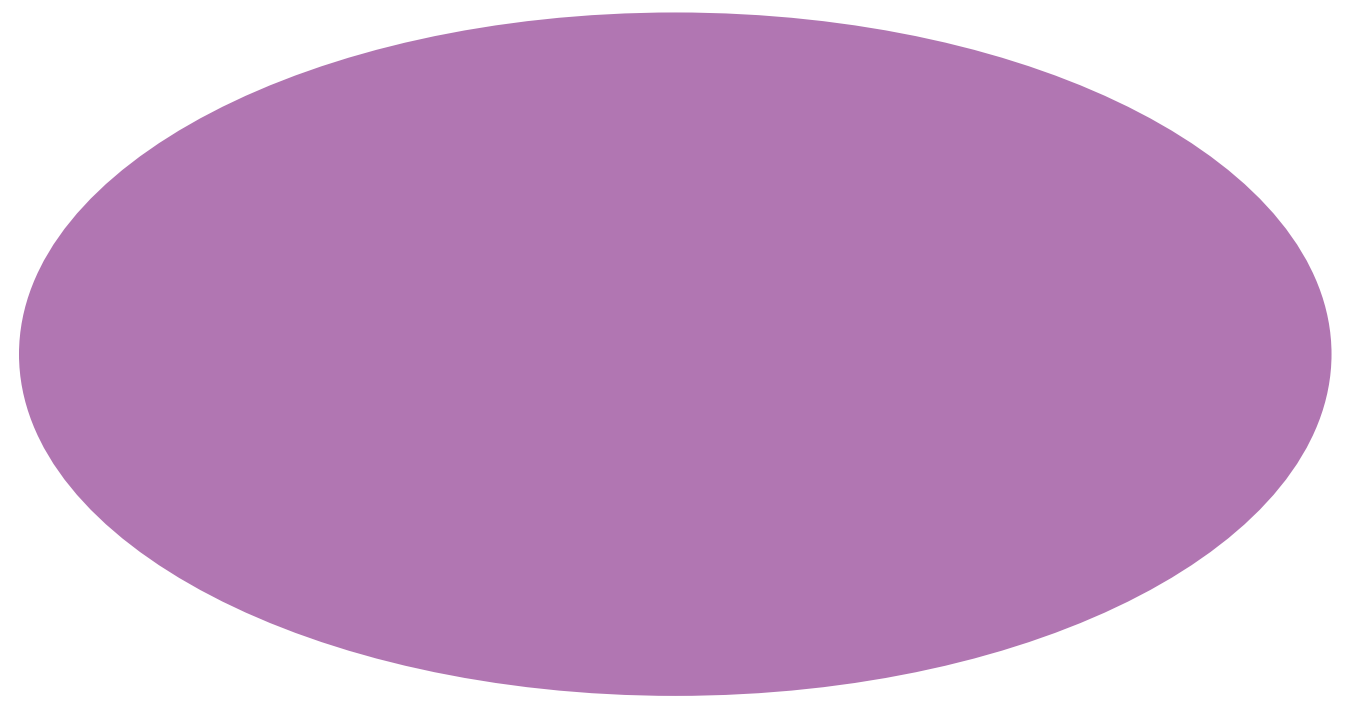

FIGURE 1. Full-sky map of the temperature of the cosmic microwave background. Variations in the temperature are a few parts in $10^{5}$ and are too small to be seen on this map.

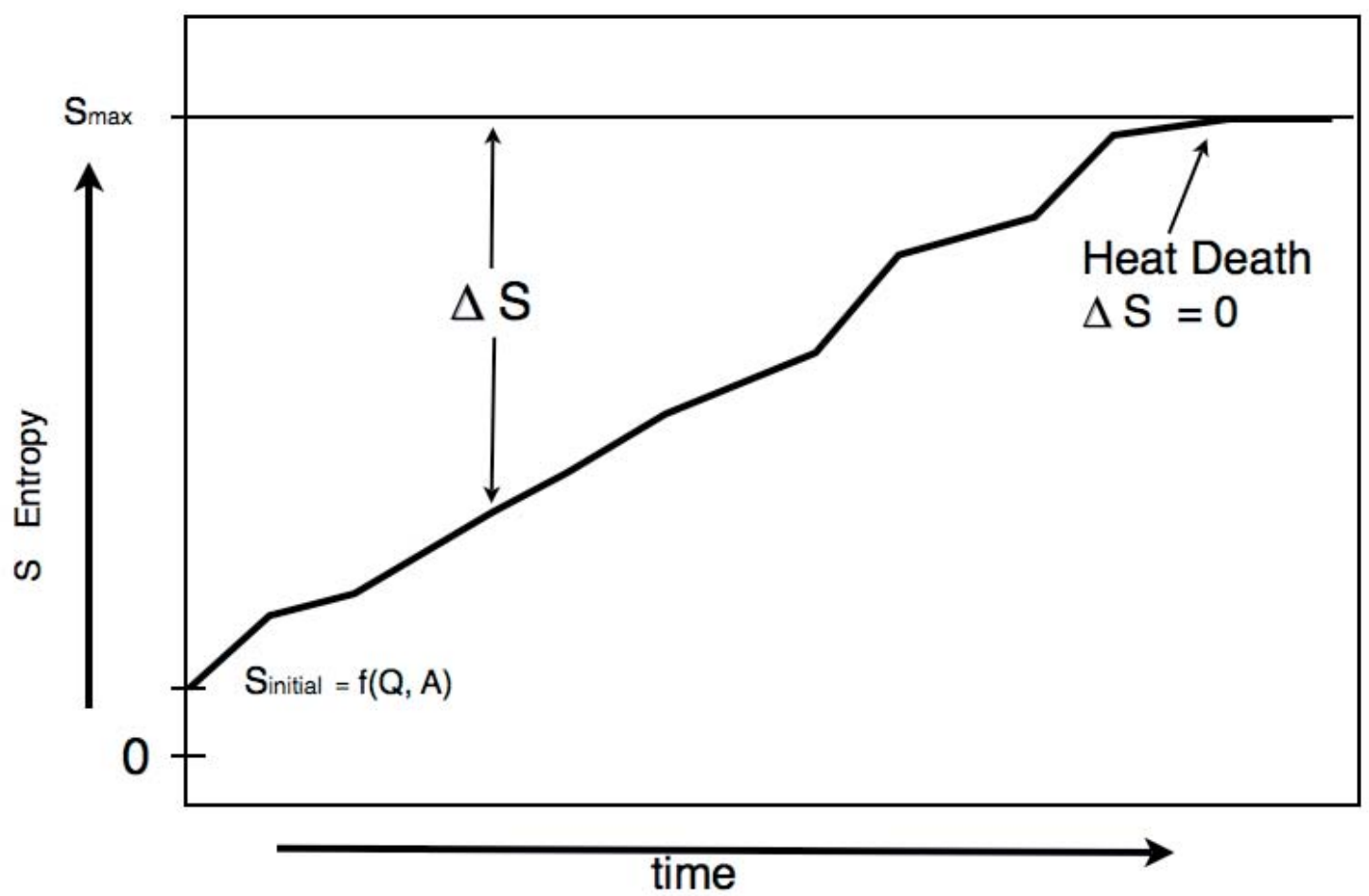

FIGURE 2. In order to satisfy $S_{\text {initial }}<<S_{\text {now }}<<S_{\max }$, and allow for the near thermal and chemical equilibrium of the $C M B, S_{\text {initial }}$ must be due to gravity and have a very low value. There is little consensus about how to define $S_{\max }$ or $S_{\text {initial }}$ or about whether $\Delta S\left(=S_{\max }-S_{\text {uni }}\right)$ is increasing or decreasing (Egan \& Lineweaver 2010). Figure from Lineweaver \& Egan (2008). 


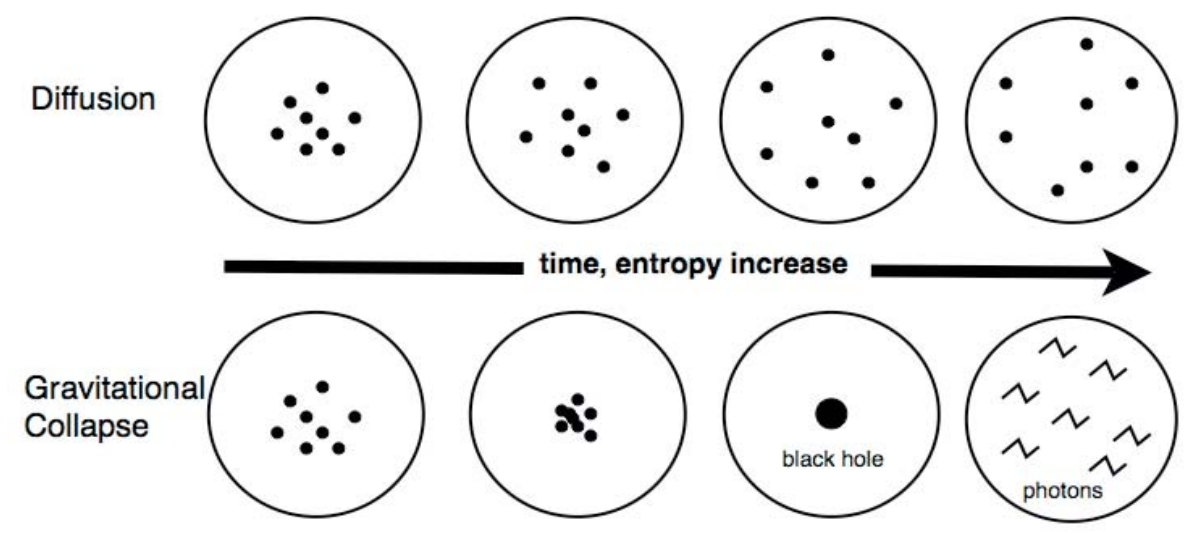

FIGURE 3. Top panel: when gravitational interactions are very weak, diffusion increases the volume and entropy of the objects (e.g. molecules of perfume in a room). Bottom panel: when the objects are large and gravitational interactions are strong, angular momentum transfer to some objects and their ejection allows the gravitational collapse of other objects into black holes. If the Hawking temperature of the black hole is higher than the environment, the black hole will evaporate as photons and other particles. In all of these irreversible processes, entropy must increase. Dark energy does not clump, so it cannot contribute to the increasing gravitational entropy of the universe. Figure from Lineweaver \& Egan (2008).

\section{PARTICLE AND EVENT HORIZONS AS NORMALIZING VOLUMES}

We would like to quantify the entropy of the universe. This can be done by considering the comoving entropy density normalized to some large representative volume (such as the current paricle horizon) or the time dependent volume inside the current event horizon. These are referred to respectively as "scheme1" and "scheme 2" in Egan and Lineweaver (2009). Here we focus on scheme 2 .

The radius of the particle horizon $R_{P H}$ is:

$$
R_{P H}(t)=a(t) \int_{0}^{t} \frac{c d t^{\prime}}{a\left(t^{\prime}\right)}
$$

Inserting the Friedmann equation for $a\left(t^{\prime}\right)$ including the dark energy term of the standard $\Lambda \mathrm{CDM}$ model, yields $R_{P H}\left(t_{o}\right)=47 \mathrm{Glyr}$. The radius of the cosmic event horizon $R_{C E H}$ is:

$$
R_{C E H}(t)=a(t) \int_{t}^{\infty} \frac{c d t^{\prime}}{a\left(t^{\prime}\right)}
$$

Inserting the Friedmann equation for $a\left(t^{\prime}\right)$ with the values of the standard $\Lambda$ CDM model yields $R_{C E H}\left(t_{o}\right)=15.7$ Glyr. If we let $t$ approach infinity we get $R_{C E H}(t \rightarrow \infty)=16.4 \mathrm{Glyr}$, which is only slightly larger than 15.7 Glyr. This can be seen in the top panel of Fig. 4 where the 15.7 Glyr and 16.4 Glyr radii of the event horizon can be read off the proper distance on the $\mathrm{x}$-axis. Notice that in comoving space (middle panel) the event horizon is shrinking and as it does, comoving galaxies move outside the event horizon.

The entropy of the cosmic event horizon according to Gibbon \& Hawking (1977) is:

$$
S_{C E H}\left(t_{0}\right)=\frac{k c^{3}}{G \hbar} \frac{A}{4}=\frac{\pi k c^{3}}{G \hbar} R_{C E H}^{2}\left(t_{0}\right)^{2}
$$



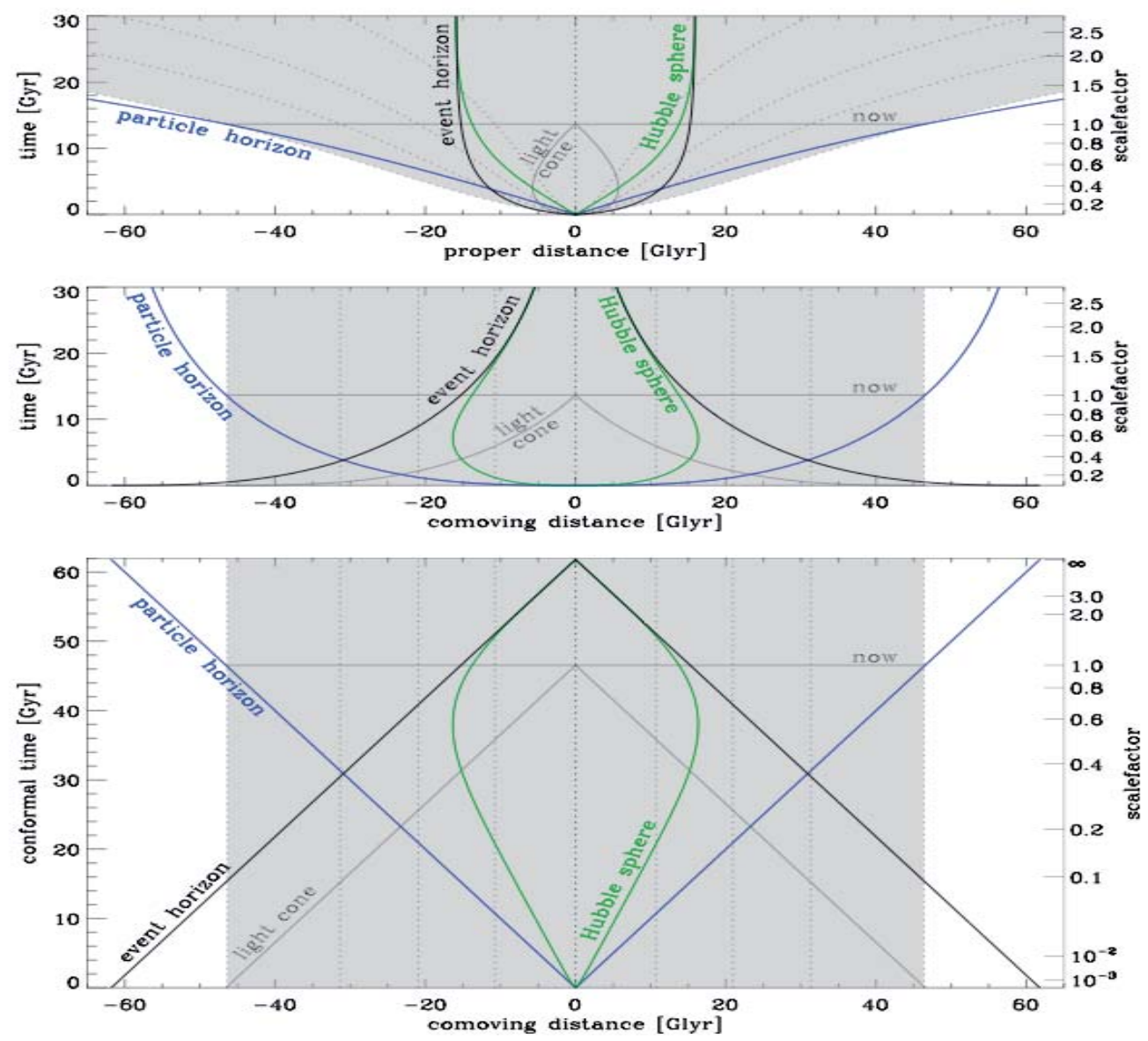

FIGURE 4. The size of the observable universe is the particle horizon (Eq. 1). The comoving size of the current particle horizon is colored grey in all three panels. The event horizon (Eq. 2) as a function of time is distinct from the Hubble sphere (where the velocity of recession equals the speed of light). In the top panel the dashed comoving lines of the galaxies show the expansion of the universe with respect to proper distance on the $\mathrm{x}$-axis. In the middle and bottom panels we use comoving space as the $\mathrm{x}$ axis, so the expansion is scaled out. In the bottom panel temporal "expansion" is scaled out by the use of conformal time = time/a.

Inserting the value for $R_{C E H}$ derived above yields:

$$
S_{C E H}=2.6 \times 10^{122} \mathrm{k}
$$

Since $\left(R_{C E H}(t \rightarrow \infty) / R_{C E H}\left(t_{o}\right)\right)^{2} \sim 1.1$, the ultimate entropy of the cosmic event horizon is only about $10 \%$ larger than the entropy associated with it today.

\section{THE ENTROPY BUDGET OF THE UNIVERSE}

We have used recent measurements of the supermassive black hole mass function to find that, after the entropy of the cosmic event horizon, supermassive black holes are the largest contributor to the entropy of the universe -- contributing at least an order of magnitude more entropy than previously estimated (Frampton \& Kephart 2008). We have also made the first tentative estimate of the entropy of dark matter within the cosmic event horizon. 
TABLE 1. The entropy of the universe including the Gibbons-Hawking entropy of the cosmic event horizon as well as the entropy of the dominant components contained within the cosmic event horizon. See Egan \& Lineweaver (2009) for details.

\begin{tabular}{|c|c|}
\hline Component & Entropy $S[k]$ \\
\hline Cosmic Event Horizon & $2.6 \pm 0.3 \times 10^{122}$ \\
\hline SMBHs & $1.2_{-0.7}^{+1.1} \times 10^{103}$ \\
\hline *Stellar BHs $\left(42-140 M_{\odot}\right)$ & $1.2 \times 10^{98_{-1.6}^{+0.8}}$ \\
\hline Stellar BHs $\left(2.5-15 M_{\odot}\right)$ & $2.2 \times 10^{96_{-1.2}^{+0.6}}$ \\
\hline Photons & $2.03 \pm 0.15 \times 10^{88}$ \\
\hline Relic Neutrinos & $1.93 \pm 0.15 \times 10^{88}$ \\
\hline Dark Matter & $6 \times 10^{86 \pm 1}$ \\
\hline Relic Gravitons & $2.3 \times 10^{86_{-3.1}^{+0.2}}$ \\
\hline ISM \& IGM & $2.7 \pm 2.1 \times 10^{80}$ \\
\hline Stars & $3.5 \pm 1.7 \times 10^{78}$ \\
\hline Total & $2.6 \pm 0.3 \times 10^{122}$ \\
\hline
\end{tabular}

\section{THE ENTROPIC MEANING OF BLACK HOLE AND COSMIC EVENT HORIZONS}

The entropy of the cosmic event horizon dominates the budget of entropy of the universe. It is unclear what significance this has. Is the Gibbons-Hawking entropy of the cosmic event horizon due to the loss of information to the observer as comoving objects go across the horizon? Davis, Davies and Lineweaver (1999) suggest that in order to obey the generalized second law, the entropy in the matter and photons that passes outside the horizon and is lost, is compensated for by the increasing entropy of the cosmic event horizon. Yet the growth of the horizon depends on the energy crossing the horizon, not the entropy. This led Bekenstein (1981) to propose the Bekenstein bound on the entropy of a system. Lloyd (2002) relates the amount of information I, in bits, to the logarithm of the number of distinct quantum states available to the system:

$I=\left(S_{\max } / k \ln 2\right)$. The monotonic growth of the cosmic event horizon (it cannot shrink because its entropy must not decrease) seems to rule-out phantom equations-of-state, where $w<-1$; these would result in a shrinking cosmic event horizon.

To interpret the $\mathrm{CEH}$ entropy itself as a maximum entropy is to invoke the holographic bound on the volume of the cosmic event horizon. Fig. 6 shows the holographic bound being violated between $10^{-40}$ and $10^{-20}$ seconds after the big bang, when the entropy of radiation is larger than the entropy of the cosmic event horizon. During this time interval the radiation within the CEH contained more entropy than the CEH itself. This happens because the holographic bound is not expected to hold on volumes much larger than the Hubble sphere. One can see in Fig. 4 that for very early times, the event horizon is much larger than the Hubble sphere. Any volume larger than the Hubble sphere is more dense than a black hole the same size. Violations of the standard holographic bound, such as the one seen here, motivated Bousso (1999) to propose a modified holographic bound, the Covariant Entropy Conjecture, which is not violated in the same way.

\section{ACKNOWLEDGMENTS}

We acknowledge useful discussions with Tamara Davis and Paul Davies. 


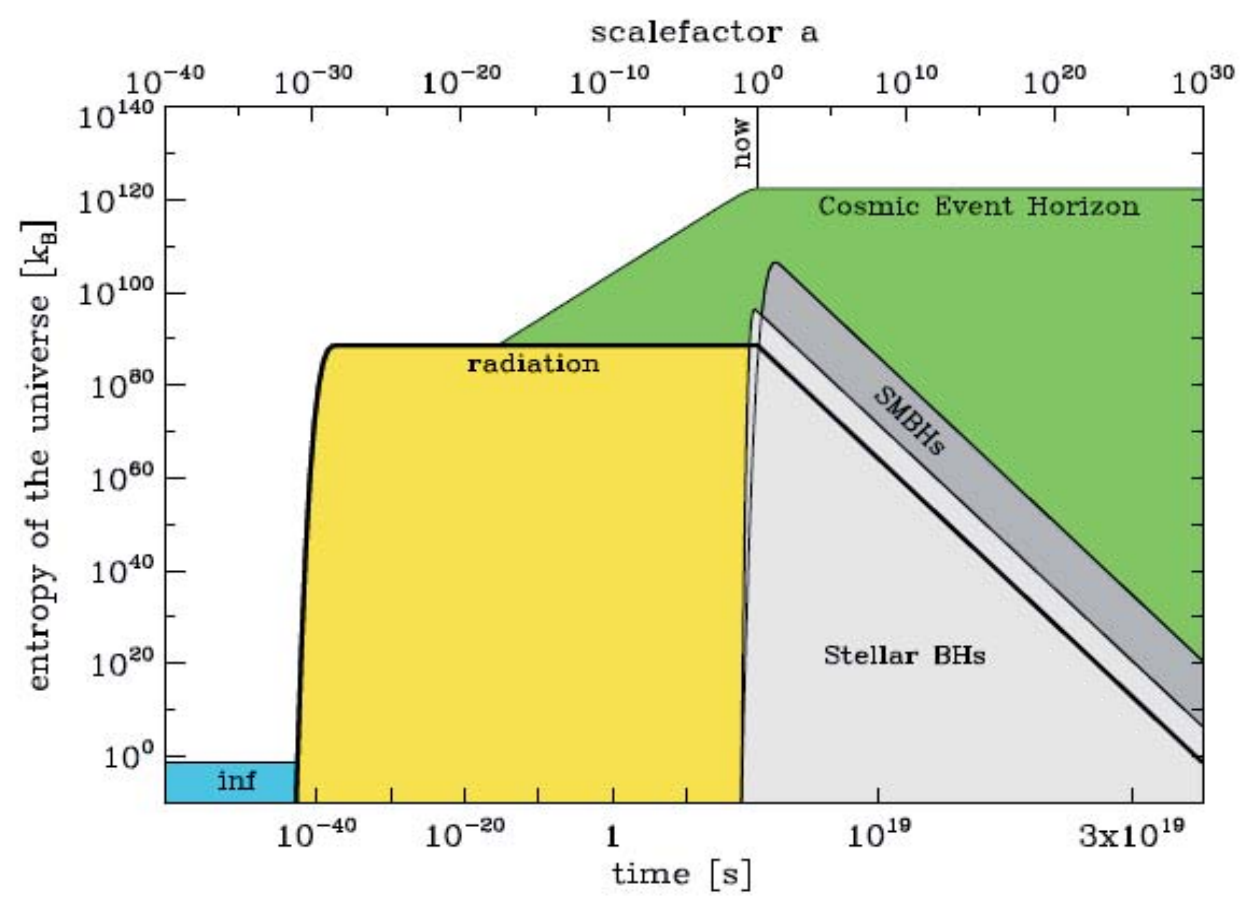

FIGURE 5. The Gibbons-Hawking entropy of the cosmic event horizon dominates the entropy budget of the contents of the event horizon. We have assumed an epoch of inflation and reheating at the Planck time, $10^{-43}$ seconds after the big bang. Although, the entropy of the event horizons of super-massive black holes ("SMBHs") is $10^{19}$ times smaller than the entropy of the current cosmic event horizon, the entropy from SMBHs dominates all other forms of entropy, including stellar mass black holes and the entropy of the cosmic microwave background and the neutrino background.

\section{REFERENCES}

1. C. H. Linewaver and C. A. Egan "Life, Gravity and the Second Law of Thermodynamics", Physics of Life Reviews, 5, 225$242(2008)$.

2. C.A. Egan and C.H. Lineweaver, "A Larger Estimate of the Entropy of the Universe", Astrophysical Journal, submitted, arXiv:0909.3983 (2009).

3. C.A. Egan and C.H. Lineweaver, "Heat Death or Eternal Life of the Universe", in preparation.

4. P.H.Frampton \& T.W.Kephart, 2008, Journal of Cosmology and Astro-Particle Physics, 6,8

5. G.W. Gibbons and S.W. Hawking "Cosmological event horizons, thermodynamics, and particle creation" Phys. Rev. D, 2738 (1977)

6. J.C. Mather, Fixsen, D.J. Shafer, R.A. Mosier, C. \& Wilkinson, D.T. 1999 Astrophysical Journal, 512, 511

7. G.F. Smoot et al 1992, Astrophysical Journal Letters, 396, 1, L1-L5

8. R. Penrose "Singularities and time-asymmetry", in General Relativity: An Einstein Centenary Survey, edited by S.W.Hawking \& W. Israel, Cambridge:Cambridge Univ. Press; 1979. p. 581-638 [Chapter 12].

9. R. Penrose "Newton, Quantum Theory and Reality", in 300 Years of Gravitation edited by S.W.Hawking \& W. Israel, Cambridge: Cambridge Univ. Press; 1987.

10. R. Penrose "Cosmology and the Arrow of Time" in The Emperor's New Mind, Oxford University Press; 1989. p. 302-47 [Chapter 7].

11. R. Penrose "The Big Bang and its Thermodynamic Legacy" in Road to Reality, London: Vintage Books; 2004. p. 686734[Chapter 27].

12. T.M. Davis, P.C.W. Davies \& C.H. Lineweaver, 2003 Class. Quantum Grav. 20 2753-2764

13. S. Lloyd, 2002, Phys. Rev. Lett 88, 237901

14. J. Bekenstein, 1981 Phys. Rev. D 23, 287 (1981)

15. R. Bousso, Journal of High Energy Physics, 7, 4 (1999) 